\title{
NEUTRINO ASTROPHYSICS WITH IMB: PAST, PRESENT, AND FUTURE
}

\author{
Presented by: \\ David W. Casper \\ Boston University, Boston MA 02215, USA and \\ The University of Michigan, Ann Arbor, MI 48109, USA \\ and \\ Lawrence R. Sulak \\ Boston University, Boston, MA 02215, USA
}

\section{IMB Collaboration:}

R.M. Bionta, G. Blewitt, C.B. Bratton, D. Casper, R. Claus, B. Cortez, M. Crouch, S.T. Dye, S. Errede, G.W. Foster, W. Gajewski, K.S. Ganezer, M. Goldhaber, T.J. Haines, T.W.Jones, D. Kielczewska, W.R. Kropp, J.G. Learned, J.M. LoSecco, J. Matthews, R. Miller, M.S. Mudan, H.S. Park, L.R. Price, F. Reines, J. Schultz, S. Seidel, E. Shumard, D. Sinclair, H.W. Sobel, J.L. Stone, L.R. Sulak, R. Svoboda, G. Thornton, J.C. van der Velde, and C. Wuest

A burst of eight neutrino interactions occurring over a six second interval has been observed with the IMB detector. The closeness in time of the burst to the optical discovery of Supernova 1987a suggests that the neutrinos originated from stellar collapse. The characteristics of the burst are reviewed together with a recently completed reevaluation of many aspects of the detector's response. Efforts underway to discover past and future supernovae are also briefly discussed.

\section{INTRODUCTION}

The IMB detector, designed to search for nucleon decay, is sensitive to other processes, such as neutrino interactions, occurring within its volume. The laboratory is located in a salt mine operated by Morton Thiokol Company near Painesville, Ohio (41.7॰ North, 81.3 ${ }^{\circ}$ West) at a depth of 1570 mwe. In its present configuration ${ }^{1}$, the detector consists of 2048 8" diameter hemispherical photo-multiplier tubes (PMT's) each mated to a $60 \times 60 \mathrm{~cm}^{2}$ wavelength-shifting plate, mounted on a 1 meter surface grid, and viewing a $23 \times 17 \times 18$ $\mathrm{m}^{3}$ cavity containing $\sim 6800$ tons of ultra-pure water. Charged particles traversing the water at speeds $\beta>0.75$ (or equivalently, $\mathrm{E}>1.5 \mathrm{~m}_{0} \mathrm{c}^{2}$ ) produce Cerenkov light, which is recorded by the PMT's. The wavelength-shifting plates also absorb light and reemit, and some of the reemitted light finds its way to the PMT/plate interface by internal reflection, approximately doubling the light collection of a bare PMT. The present IMB detector is four or five times as sensitive as the original design ${ }^{2}$.

When about 20 PMTs (whose discriminators are set near the $1 / 4$ photo-electron level for high efficiency) are fired within $55 \mathrm{~ns}$, the detector is triggered and the data from the event is written on computer tape for subsequent analysis. Approximately 250,000 triggers occur each day, almost all due to cosmic ray muons penetrating from the surface. For each PMT which is fired in an event, the time of firing and number of photo-electrons (pe's) is recorded. A late time scale is also available to record light produced by electrons from any 
muon decay following the trigger. In addition to the data from the PMT's, the absolute time of the trigger is recorded from a WWV clock, which at the time of the observed burst had an accuracy of $\pm 50 \mathrm{~ms}$; the relative times of the events are accurate to $\pm 0.5 \mathrm{~ms}$. Finally, the amount of time the detector is dead following each trigger (which averages about $35 \mathrm{~ms}$ ) is also measured.

The timing, geometry, and pulse-heights of the PMT's fired are used off-line to reconstruct the location of the event vertex and the direction of the interaction product(s). The interaction vertex can be determined to within about 1 meter, and the direction of low-energy tracks to within about $20^{\circ}$ when their $41^{\circ}$ half-angle Cerenkov cone patterns do not overlap. For nearly massless secondaries like $\mathrm{e}^{+}$or $\mathrm{e}^{-}$, the particle energies can be simply determined from the pulse-heights of the illuminated PMT's. This process is slightly complicated by Cerenkov threshold effects for more massive particles such as muons. The conversion from pe's to energy has been achieved by analyzing samples of through-going cosmic ray muons and low-energy electrons produced by the decay of muons which stop in the detector. The differences between the two methods give an estimate of the systematic error in the energy measurement, which we quote as $\pm 10 \%$. In addition, for low energy events, the statistical uncertainty of counting just a few dozen photo-electrons must also be considered, and in this case, dominates. Finally, non-exiting muons can be identified by a late coincidence of hits produced by their decay, with an efficiency of $73 \%$ for $\mu^{+}$and $60 \%$ for $\mu^{-}{ }^{3}$. In the absence of an identified muon decay, particle identification is unreliable with existing methods.

\section{Neutrinos From Stellar Collapse}

The gravitational collapse of a massive star is followed by the release of the binding energy ( $\sim$ few $\times 10^{\text {ss }}$ ergs) of the resulting neutron star ${ }^{4}$. The most effective mechanism for transporting this energy away from the star is the radiation of neutrinos and anti-neutrinos (of all flavors) produced by $\mathrm{e}^{+} \mathrm{e}^{-}$annihilation and weak bremstrahlung within the collapsed core. Substantially less energy ( $\mathrm{a}$ few $\times 10^{52} \mathrm{ergs}$ ) is carried away by $\nu_{\varepsilon}$ from electron capture during neutron star formation ("deleptonization"). The length of the two neutrino pulses is determined by the diffusion of neutrinos through the rapidly compressing and heating, then rapidly expanding and cooling core/neutron star. Most authors agreed even before SN1987a that the $\nu_{a}$ rich deleptonization pulse should last about $100 \mathrm{~ms}$, while the thermal radiation of neutrinos of all flavors should last on the order of seconds ${ }^{\mathbf{s}}$. The validity of a Fermi-Dirac distribution with a single temperature as a description of the neutrino energy spectrum is not universally accepted, but in this approximation, temperatures of 3-5 $\mathrm{MeV}$ are expected. The corresponding mean neutrino energies are then $10-15 \mathrm{MeV}^{\circ}$.

At these energies, the $\bar{\nu}_{\varepsilon}$ component of the flux is the most easily detected in water Cerenkov detectors, due to the relatively large inverse beta decay $\left(\overline{\nu_{e}}+p \rightarrow e^{+}+n\right)$ crosssection and the number of available of free protons in such devices. Scattering off electrons and oxygen nucleii should be much more rare. The total cross-sections (summed over the appropriate number of scatterers) for the 6800 ton IMB detector are shown in Figure 1 ?

\section{The 23 February, 1987 Neutrino Burst}

On 23 February, 1987, in a six-second interval beginning at 7:35:41.37 \pm .05 (universal 
time), nine events with less than $\mathbf{7 0}$ hits were recorded by the IMB detector. Off-line analysis determined that eight of these nine events were indeed produced by relativistic charged particles originating inside the detector, while the remaining event was due to a cosmic ray muon clipping one corner of the apparatus. A search of some sixty hours of data recorded before and after the burst revealed no other occurrence of more than 5 such triggers in any 10 second interval, with the coincidence multiplicity fitting a Poisson distribution with a mean of 0.8 triggers/ 10 second interval. All these other low light-level events are consistent with cosmic ray muons, and none originate inside the detector. The eight events in the burst all appear qualitatively similar to low-energy electrons produced by muon decays. None of the eight events themselves have an associated muon decay. The events are distributed throughout the detector volume, as are atmospheric neutrino interactions. We therefore identify the eight events as the products of low-energy neutrino interactions within the detector. Assuming roughly equal fluxes of all $\nu$ and $\bar{\nu}$, cross-sectional arguments (see above) further suggest the events are positrons from the reaction $\overline{\nu_{\varepsilon}}+p \rightarrow e^{+}+n$, although other possibilities do exist ${ }^{8}$. The incident neutrino energies inferred from the visible electron energies under this hypothesis, the angles of the reconstructed positron tracks with respect to the direction of the supernova, and the times of the eight events are summarized in Table 1.

Neutrino interactions are recorded at the rate of about one per day at all energies, and about one per seven days with visible energies below $100 \mathrm{MeV}$. Thus, the liklihood of the eight events in six seconds being a statistical fluctuation of the ambient rate is enormously smalle. Due to the temporal proximity of the burst to the appearance of $S N 1987 \mathrm{a}^{10}$, the inability of any other known process to create such a short and intense burst of comparable energy neutrinos, and the simultaneous observation of a similar burst in the Kamiokande II experiment ${ }^{11}$, it is reasonable to conclude that the neutrino burst originated with the gravitational collapse of SN1987a.

The triggering efficiency of the $\mathbf{6 8 0 0}$ ton detector as a function of energy, determined by mimicking low-energy particle tracks with a laser-driven hardware simulator and with computer Monte Carlos, is shown in Figure 2. In both cases, the PMT's affected by a malfunctioning power supply (see below) were ignored to reproduce the state of the detector at the time of the burst. These efficiencies for the 6800 ton volume are lower than those previously published ${ }^{12}$ for a 5000 ton central sub-volume, as would be expected. The use of the full 6800 ton mass is justified by the fact that one of the eight events does occur very near the detector's surface. The observed (but not total) inefficiency near the detector's edges is thus correctly accounted for by averaging over the entire volume.

Correcting the observed events for efficiency means about $32 \pm 15$ interactions with $E \geq \sim$ $20 \mathrm{MeV}$ occurred inside the detector, with the spectrum of energies shown in Figure 3a. Correcting for the cross-section for inverse beta decay, the incident neutrino spectrum is shown in Figure 3b, with a Fermi-Dirac distribution at $\mathrm{T}=4.2 \mathrm{MeV}$ also shown for comparison. The total time-integrated flux of $\overline{\nu_{a}}$ is then $(0.8 \pm 0.3) \times 10^{10}$ neutrinos $/ \mathrm{cm}^{2}$, corresponding to a total energy release of $(4.8 \pm 1.7) \times 10^{52}$ ergs in $\overline{\nu_{c}}$ at the $49 \pm(5-10) \mathrm{kpc}$ distant source.

If the observed neutrino interactions are due to the inverse beta decay reaction, the angular distribution of the positrons with respect to the direction of the supernova should 


\section{TABLE 1}

Event Number Time (UT)

33162

33164

33167

33168

33170

33173

33179

33184

\section{Neutrino Energy (MeV)}

$40 \pm 7$

$39 \pm 7$

$30 \pm 6$

$41 \pm 7$

$38 \pm 9$

$38 \pm 6$

$21 \pm 5$

$24 \pm 5$

\section{Angle (degrees)}

$80 \pm 10$

$43 \pm 15$

$56 \pm 20$

$66 \pm 20$

$32 \pm 15$

$52 \pm 10$

$40 \pm 20$

$104 \pm 20$

\section{Explanations:}

Event Number: Number of the event on the data tape. Event numbers are not sequential because cosmic ray muons are interspersed with the neutrino events.

Time:

Absolute times are accurate to $\pm 50 \mathrm{~ms}$. Relative times are accurate to $\pm 0.5 \mathrm{~ms}$.

Neutrino Energy: Neutrino energies under the inverse beta decay hypothesis. Measured particle kinetic energies are $1.8 \mathrm{MeV}$ less. Error quoted is statistical only. Systematic error estimated to be $\pm 10 \%$

Angle:

Angle of observed track to the direction from the supernova.

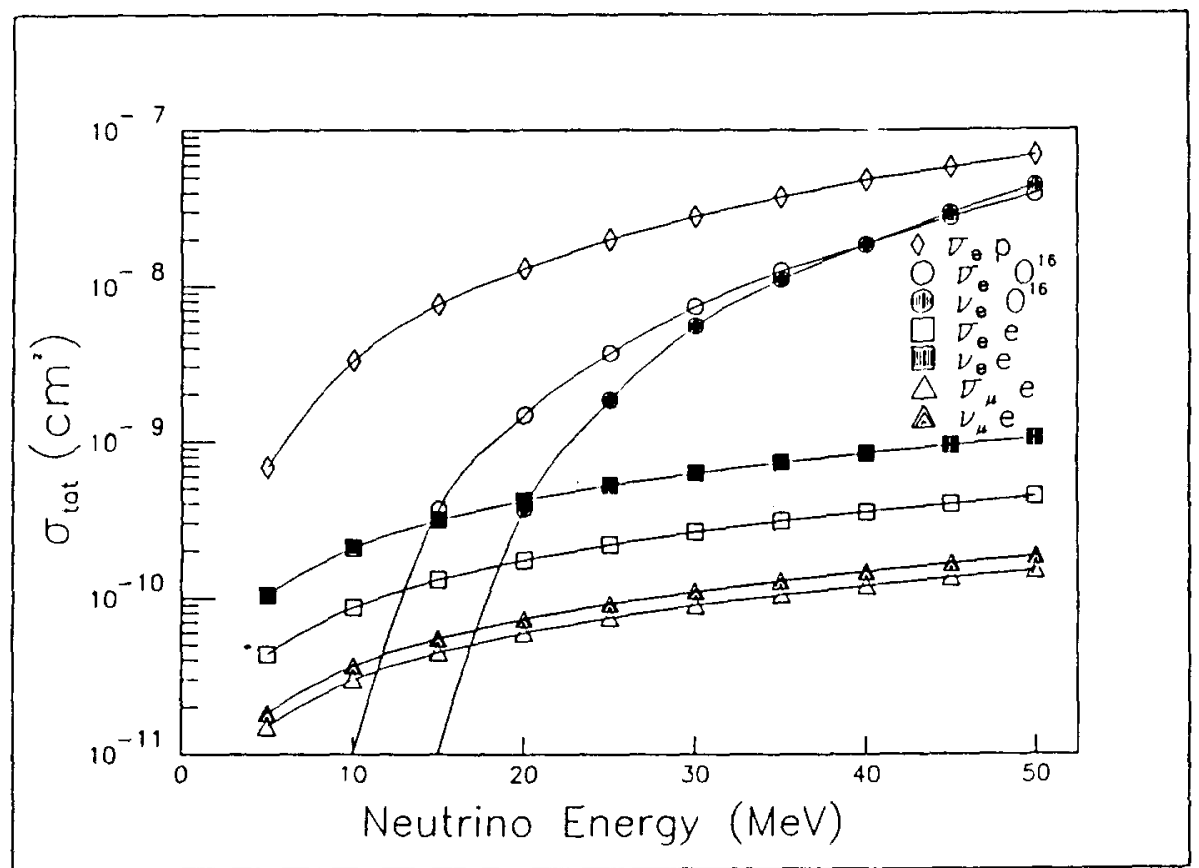

FIGURE 1: TOTAL CROSS-SECTIONS OF VISIBLE NEUTRINO PROCESSES FOR 6800 TON IMB DETECTOR 


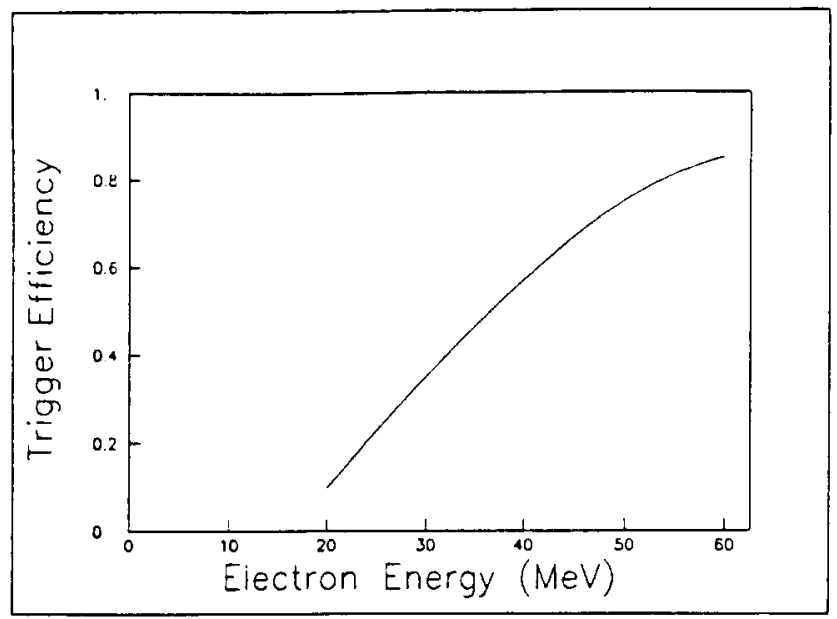

FIGURE 2: TRIGGERING EFFICIENCY FOR 6800 TON IMB DETECTOR



FIGURE 3a: EFFICIENCY CORRECTED SPECTRUM OF INTERACTIONS (NORMALIZED TO 1 KTON)

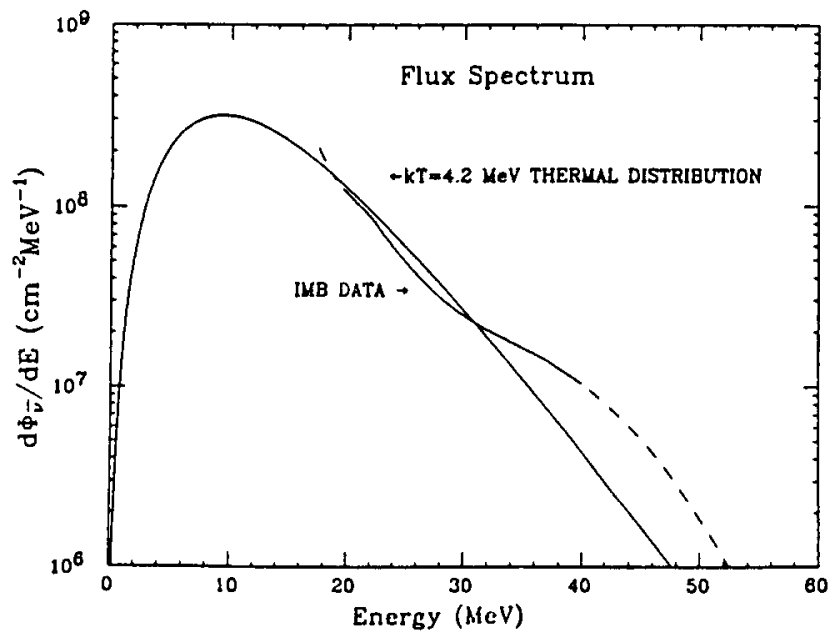

FIGURE 3b: CROSS-SECTION CORRECTED SPECTRUM OF INCIDENT NEUTRINOS (WITH FERMI SPECTRUM FOR COMPARISON) 
be isotropic to within $\sim 10 \%$. Unfortunately, directional analysis of the data is complicated by the fact that one nearly contiguous region of the detector was rendered inoperative by the failure of one of four power supplies some two hours before the arrival of the neutrino pulse. A detailed study of this problem is presented elsewhere ${ }^{13}$, but here it is worth noting that seven of the eight events point in the forward hemisphere from the supernova direction, while only one points in the backward hemisphere. Nonetheless, when smeared over the reconstruction uncertainties, the probability of obtaining such polar angles from an isotropic parent distribution is $20 \%$ under the Kolmogorov-Smirnov test, even if no possible instrumental biases are taken into account.

\section{Search For Past and Future Neutrino Bursts}

Knowing the characteristics of the observed burst of neutrinos from stellar collapse makes it much easier to search for other such phenomena. Data collected since the detector was turned on in September 1982 has been analyzed to find low-energy events occurring in rapid succession like those of the 1987 burst.

Data comprising 335 live-days during the initial run with 5" PMTs contained no 3 second period with 6 or more low-energy triggers which could not be identified as sick PMTs which become light sources. Such failures are easily excluded from the analysis since they typically last for minutes or hours (until quelled by the operator removing power from them) and create hit patterns with diffuse timing and nearly identical geometry event after event. Using the 1987 burst for reference, the search criteria are determined to be $100 \%$ percent efficient for finding collapses occurring within $20 \mathrm{kpc}$ of Earth, a range containing $95 \%$ of the stars in our galaxy ${ }^{14}$.

140 days of data recorded during the present detector run but prior to SN1987a have also been analyzed. Again, no occurrences of 6 or more contained events within 10 seconds were uncovered ${ }^{15}$. This search should also be $100 \%$ efficient at ranges up to $20 \mathrm{kpc}$. Since shortly after SN1987a (67 live-days to present), real-time algorithms have been applied to the data. These algorithms notify the on-site operator when seven events with $E<\sim 100$ $\mathrm{MeV}$ are recorded within 10 seconds. In addition, all low-energy events are subjected to a vertex finding routine, and the operator is notified whenever 4 events in 10 seconds are reconstructed 2 or more meters from the detector walls. This analysis should also find all stellar collapses within a $20 \mathrm{kpc}$ radius, and we note that SN1987a, occurring at a distance of $49 \mathrm{kpc}$, would have satisfied both of the above criteria.

In total, 442 days of data have been analyzed. Except for the burst on 23 February, 1987, no evidence for stellar collapse was found. This gives a $90 \% \mathrm{CL}$ limit on the number of stellar collapses within $20 \mathrm{kpc}$ of $<1.55$ / year.

Observation of neutrinos from SN1987a has already resulted in interesting limits on the mass ${ }^{10}$, lifetime ${ }^{17}$, and magnetic moment ${ }^{18}$ of $\overline{\nu_{e}}$ as well as the number of light neutrino species ${ }^{10}$, based on the detection of two dozen or less events world-wide. A collapse occurring within our galaxy (range $\sim 10 \mathrm{kpc}$ ) should produce about 25 times as many interactions as $1987 \mathrm{a}$, and could provide enormous improvements to the above limits, as well as strongly constraining (or even measuring) the mass of $\nu_{\mu}$ and perhaps $\nu_{r}$ if neutral current scattering off electrons could be convincingly observed. The IMB Collaboration has undertaken an 
effort to improve the detector's ability to discover neutrinos from a nearby supernova. In addition to augmenting the detector's reliability, and hence maximizing the amount of uptime during which a burst can be recorded, it will also be necessary to increase the maximum data collection rate, since the expected signal ( 200 events in a few seconds) would presently swamp the detector and many events would be lost. With these improvements, IMB hopes to continue to play a leading role in the exciting new age of neutrino astronomy.

\section{Acknowledgements}

Many thanks to the organizers for their work in producing a suberb conference. Also thanks to S. Nussinov and T. Walker for helpful advice on this manuscript. The IMB Collaboration also acknowledges the hospitality of the Morton-Thiokol company for providing facilities and assistance throughout the experiment. This work supported in part by grants from the United States Department of Energy.

\section{References and Notes}

1. For a full description of the IMB-3 detector with 8" PMTs, see:

D. Casper, Observation of Neutrinos From Supernova 1987A, University of Michigan Ph.

D. Thesis, (to be published).

2. For descriptions of the original IMB-1 detector with 5" PMTs, see:

R.M. Bionta, et al., Phys. Rev. Lett., $\underline{51}, 27$ (1983).

B.G. Cortez, et al., Phys. Rev. Lett., $\underline{52}, 1092$ (1984).

3. This efficiency includes losses from $\mu^{-}$absorption in water.

4. S.A. Colgate and R.H. White. Ap. J., 143, 626 (1966).

J.R. Wilson, et al., Ann N.Y. Acad. Sci., 470, 267 (1986).

5. T.J. Mazurek, Nature, 252, 287 (1974).

A. Burrows, et al., Ap. J., 251, 325 (1981).

R.F. Sawyer and A. Soni, Ap. J., 230, 859 (1979).

6. D. Schramm and W.D. Arnett, Ap. J., $\underline{198}, 629$ (1974).

R.L. Bowers and J.R. Wilson, Ap. J. Suppl., $\underline{50}, 115$ (1982).

7. C. Longuemar, " $\nu / \bar{\nu}$ Cross-sections at Low Energy Revisited", University of Michigan Research Note (May,1987), and references therein.

8. For instance, charged current interactions of $105<\mathrm{E}<\sim 250 \mathrm{MeV} \nu_{\mu}$ producing muons below Cerenkov radiating threshold, with the decay electrons producing the observed signal. All alternate scenarios require an enormous increase in the radiated energy, and an explanation of why $\bar{\nu}_{\mathrm{e}}$ interactions are missing.

9. Based on Poisson statistics, such a fluctuation should occur once every $\sim 10^{34}$ years $(\sim$ $\tau_{\text {proton!) }}$

10. I. Shelton, IAU Circular No. 4316. 
11. K. Hirata, et al., Phys. Rev. Lett., 묘, 1490 (1987).

12. R.M. Bionta, et al., Phys. Rev. Lett., $\underline{58}, 1494$ (1987).

13. C.B. Bratton, et al., "Angular Distribution of Events From SN1987a", University of Michigan preprint (1987).

14. C.B. Bratton, et al., in Proceedings of the XXth International Cosmic Ray Conference, Moscow 1987 (to be published).

15. Although the criterion for a burst has been set at 6 (or 4 , or 7 ) events in 10 seconds, the particular choice has only been made so high for ease and automation of analysis. In fact, no more than one event truly believed to originate inside the detector has ever been observed in any 10 second period, except on 23 February, 1987.

16. A burst of countless papers on limits to the neutrino mass appeared shortly after the neutrinos from the supernova. For a useful discussion of the model dependence of these limits and a partial list of mass limit references, see:

L.F. Abbott, A. De Rujula, and T.P. Walker, " Constraints on the Neutrino Mass From the Supernova Data: A Systematic Analysis", Boston University Preprint 87-24 (submitted to Nucl. Phys. B, 1987).

17. A simple limit of $\gamma \tau>1.6 \times 10^{8}$ years can be achieved based on the distance to the star.

18. I. Goldman, Y. Aharonov, G. Alexander, and S. Nussinov, Tel-Aviv University Preprint (1987).

19. D.N. Schramm, Proceedings of XXIInd Recontre de Moriond, in press (1987). 